\title{
Tracheal stricture following tracheostomy
}

\author{
P. B. D E V E R A L L
}

From Killingbeck Hospital, Leeds, 14

\begin{abstract}
Six cases of tracheal obstruction following tracheostomy are described in which surgical intervention was necessary. These occurred in a series of 104 tracheostomies. In adults the strictures have occurred at the site of pressure of the tracheostomy tube cuff. Management has been by excision of the stricture with end-to-end reconstruction. Tracheal narrowing in children has been of a different type, occurring at stomal level, and management has been more difficult.
\end{abstract}

Experience with tracheostomy has been obtained in this unit mainly with post-operative open-heart cases and chest injuries, and in all age groups except the neonate. It has been used whenever assisted ventilation has been necessary for longer than 24 hours. Tracheostomy in this group of patients has been used for intervals of from two to three days up to six weeks. Complications have been tube displacement, blockage, tracheal stricture, tracheal perforation, haemorrhage, tracheooesophageal fistula, and infection. The latter has been with a variety of organisms, but in the last two years Pseudomonas pyocyanea infection has become increasingly common and has proved very difficult to treat except when associated with removal of the tracheostomy tube. Meade (1961) records one case of tracheal stenosis in 212 cases. Putney (1955) describes the condition in two children, while Watts (1963) commented on the rarity of this complication. More recently, Gibson (1967) and Johnston, Wright, and Hercus (1967) have published papers stressing the greater incidence of tracheal damage and stricture following tracheostomy. Fraser and Bell (1967) have also described a case of stricture occurring at the site where previous tracheostomy tube cuff pressure had been present.

This paper describes and discusses six examples of patients with tracheal stricture occurring in a series of 104 tracheostomies. The mortality in this series resulting directly from tracheostomy complications is $3 \%$. A further subglottic stricture, which developed in a patient in whom the cricoid ring and first tracheal ring were damaged, is excluded.

\section{CLINICAL EXPERIENCE}

CASE 1 A 50-year-old man suffered chest injuries following a road accident in April 1962 and required assisted ventilation, a tracheostomy tube being in position for three weeks. Slight stridor was first noticed about one month later, but the symptoms only became severe in March 1963. A tracheogram showed a stricture at the level of the manubrium sterni. At operation in April 1963 a $1 \mathrm{~cm}$. long stricture was found involving the whole circumference of the trachea and corresponding to the position of the cuff of a tracheostomy tube in position. The stoma site was marked only by scar tissue anterior to the trachea. The stricture was resected and end-to-end anastomosis was performed. There were no post-operative complications and a bronchoscopy in October 1965 showed a normal tracheal lumen.

CASE 2 A 36-year-old woman suffered chest injuries following a road accident in November 1962 and required assisted ventilation, a tracheostomy tube being in position for four weeks. Towards the end of this period difficulty was experienced with tracheal suction, and on viewing the trachea with a bronchoscope granulation tissue was found at the site of the tip of the tracheostomy tube. This tissue was removed with nibbling forceps. The patient remained well after discharge until April 1963, when she complained of 'difficult breathing and breathlessness'. A tracheogram (Fig. 1) showed a moderately tight stricture. In May 1964 she complained of stridor and operation was carried out. The stricture was $2 \mathrm{~cm}$. in length and circumferential. Its position corresponded to the site of the cuff of the tube. Resection and end-to-end anastomosis was possible and she did well. A further tracheogram in September 1964 (Fig. 2) showed some narrowing at the suture line, but the patient has remained free of symptoms.

CASE 3 A 22-year-old woman had a closed mitral valvotomy in 1957 and this was repeated in 1963. She failed to achieve the expected benefit and further investigation revealed tricuspid stenosis. This was relieved by open operation with extracorporeal 

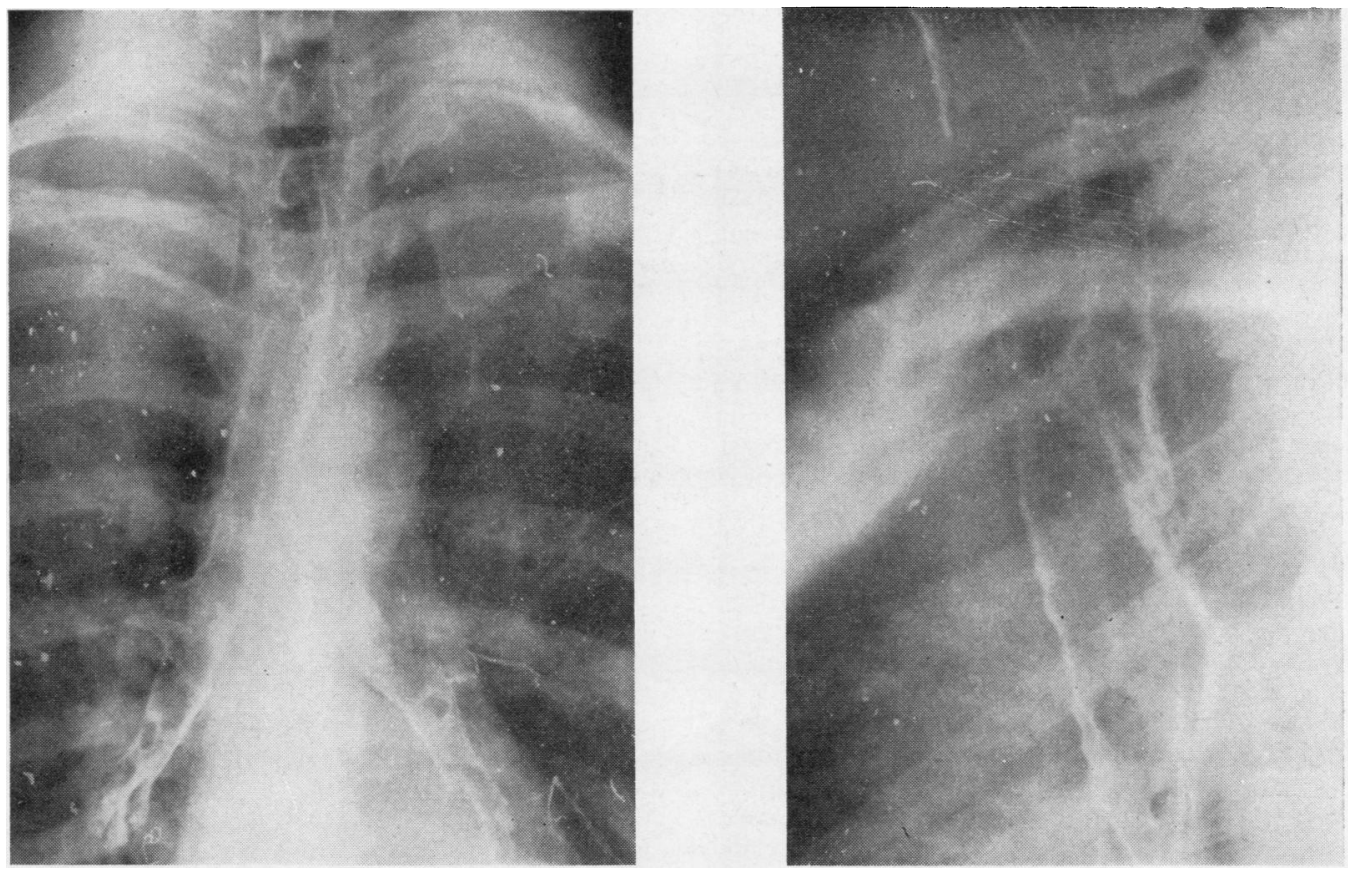

FIG. 1. Case 2 (left and right) Pre-operative tracheogram.
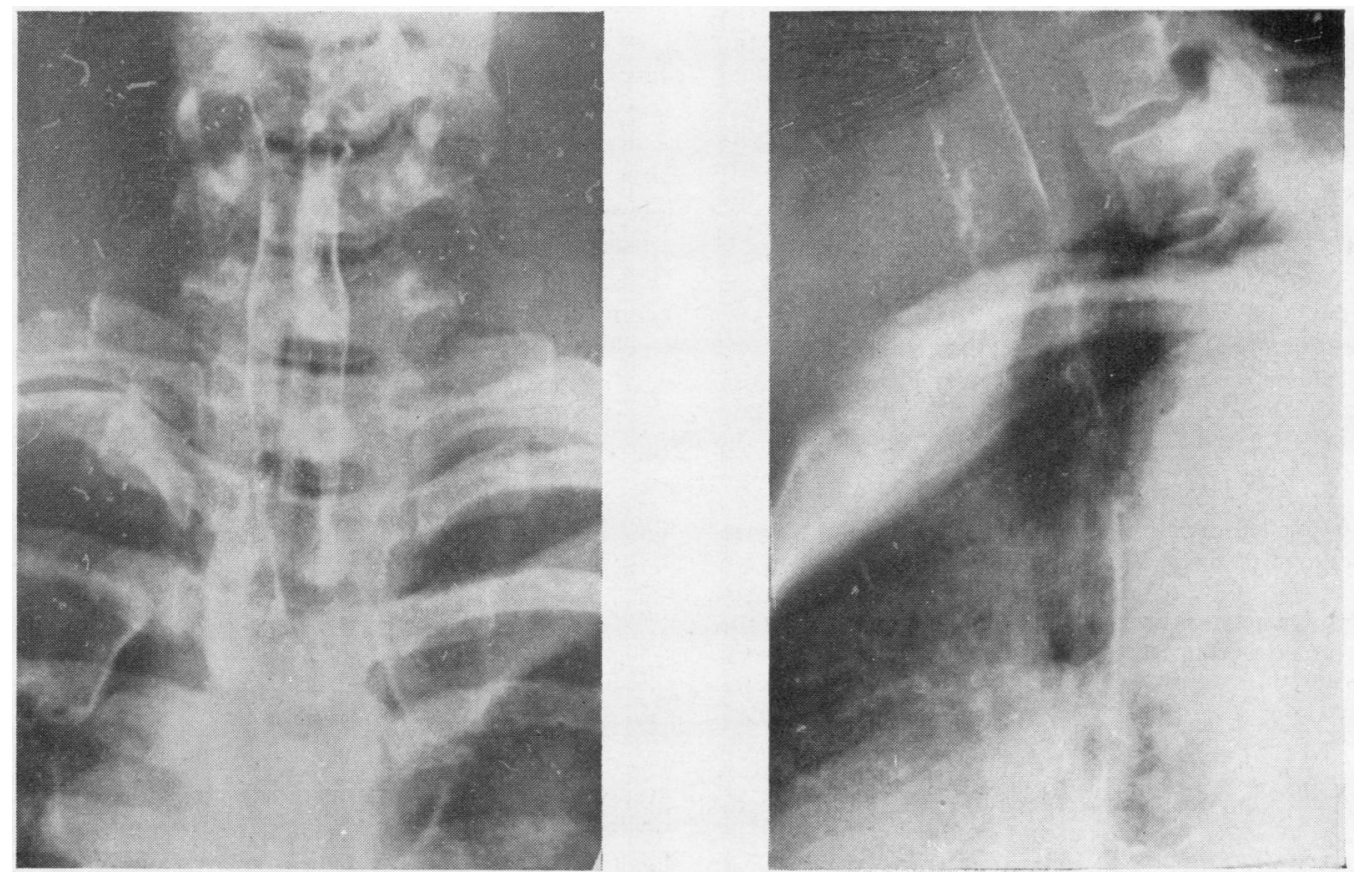

FIG. 2. Case 2 (left and right) Post-operative tracheogram. 
perfusion in November 1964. On the second postoperative day she developed pulmonary oedema, and positive pressure ventilation with a tracheostomy was needed for 10 days. In February 1965 she was re-admitted with pneumonia and severe breathlessness. A bronchoscopy showed a tight tracheal stricture. At operation in March 1965 there was a stricture at the former cuff site, and resection with end-to-end anastomosis was possible. She has remained well.

CASE 4 A 12-year-old girl with Fallot's tetralogy had a total correction performed in January 1966. Positive pressure ventilation was necessary post-operatively. A tracheostomy tube was in position for 16 days. One week after removal of the tube severe stridor was evident and bronchoscopy revealed a tight stricture with much granulation tissue at the stoma level. Immediate excision and end-to-end anastomosis was attempted, but further obstruction with granulation tissue necessitated the re-creation of a tracheostomy through the same site. In June 1966 a further resection was performed. On this occasion it was decided to leave a nasotracheal tube in position. All was well until five days later, when tube blockage with cardiac arrest occurred. Resuscitation was not successful.

At necropsy the tracheal anastomosis was satisfactory, but further tracheal damage and narrowing was present at a site corresponding to the tip of previous intratracheal tubes.

CASE 5 A 4-year-old girl had an operation for aortic coarctation with a patent ductus arteriosus in May 1966. Post-operatively she developed severe secretional airway obstruction and a tracheostomy was created. The tube was in position for nine days. After only a few days stridor became evident. Bronchoscopy revealed the trachea to be angled forwards at the stoma site and narrowed by granulation tissue. It was possible to pass a nasotracheal tube across this area, but the child suffered a fatal secondary haemorrhage from the aortic suture line some three days later.

Post-mortem examination of the trachea showed severe distortion at the tracheal stoma site with concentric granulation tissue formation and the angling previously described.

CASE 6 A 45-year-old woman had a mitral valve replacement using a $3 \mathrm{M}$ Starr Edwards prosthesis on 28 November 1966. Assisted ventilation was necessary for 10 days post-operatively and a tracheostomy tube was in position for one day longer. She recovered well and went home on 7 January 1967. She was readmitted on 16 January 1967 following the sudden onset of breathlessness with stridor. Bronchoscopy on the following day revealed a tight tracheal stricture at the cuff site. Immediate excision (Fig. 3) with endto-end anastomosis was carried out and the patient has done well. There was some hoarseness of voice for two weeks after operation.

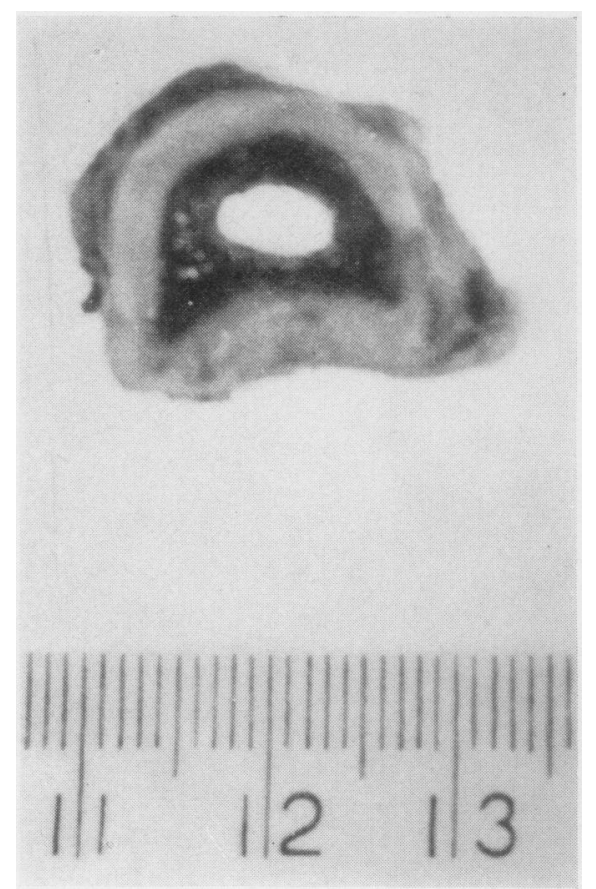

FIG 3. Case 6. Tracheal stricture excised.

DIAGNOSIS

The possibility of tracheal stricture should be considered in any patient who previously has had a tracheostomy. Stridor is commonly but not always present. Patients may complain of breathlessness or difficult breathing. We have seen an episode of pneumonia as the first presenting feature. In children, stridor is usual, but we have seen two patients with a proved slight degree of stricture not as yet justifying surgery in whom a dry repetitive cough was the complaint: in both of them a rising respiratory rate was present before the onset of stridor, soon after removal of the tube. If suspected, confirmation of the diagnosis is obtained by a tracheogram and/or bronchoscopy.

\section{OPERATION}

In all the cases described it has been possible to perform the operation through the neck. We have as yet no experience of intrathoracic tracheal stricture requiring an approach through the chest.

General anaesthetic is administered through an endotracheal tube sited above the stricture. No attempt should be made to dilate the stricture or 
to pass an endotracheal tube through it. The stricture is exposed through a cervical collar incision, the trachea being mobilized inside its fascial coat as far down into the mediastinum as is possible. The site of the stricture is usually obvious, palpation revealing an area of thickening of the tracheal wall. The trachea is then opened below the stricture, the lumen being swabbed for bacteriological-examination, and then a sterile cuffed endotracheal tube is introduced into the lower trachea through the wound for continuation of the anaesthetic. A further tracheal incision above the stricture permits this to be examined and the degree of involvement of the trachea estimated. If the stricture be circumferential resection of this segment and end-to-end anastomosis using $3 / 0$ everting silk sutures is performed, first suturing the posterior wall. Tissue forceps are used to secure and lift the lower segment. An assistant approximates the tracheal ends during suture, tying to avoid tension. A trans-oral endotracheal tube is then reintroduced and the anterior tracheal suture line is completed. It may be tempting, if only the anterior part of the trachea is involved, to wedge resect this portion and then resuture the trachea. There is a tendency with this manœuvre to cause puckering of the posterior membranous tracheal wall, so reproducing narrowing. If possible the suture line is covered with the thyroid isthmus and the neck incision is closed without drainage. Haemostasis is vital.

Post-operatively no endotracheal tube is used. The patient is nursed in an atmosphere of high humidity, and appropriate antibiotics are administered.

\section{DISCUSSION}

The indications for, management of, and complications following tracheostomy have been discussed by Björk and Engström (1955), Watts (1963), and Clarke (1965). Watts quotes a mortality directly related to the creation of a tracheostomy of $2 \%$. We have three such deaths in a series of 104 cases-case 4 in this paper, a woman who developed a tracheo-oesophageal fistula, and another patient who died from haemorrhage following perforation of the anterior tracheal wall by the tip of a silver tube. This type of tube is no longer used.

The technique of the operation of tracheostomy varies, but in this series the flap technique (Fig. 4) (Björk and Engström, 1955) has been used with attachment of the tracheal flap to the superficial fascia and lower skin edge. A vertical skin incision
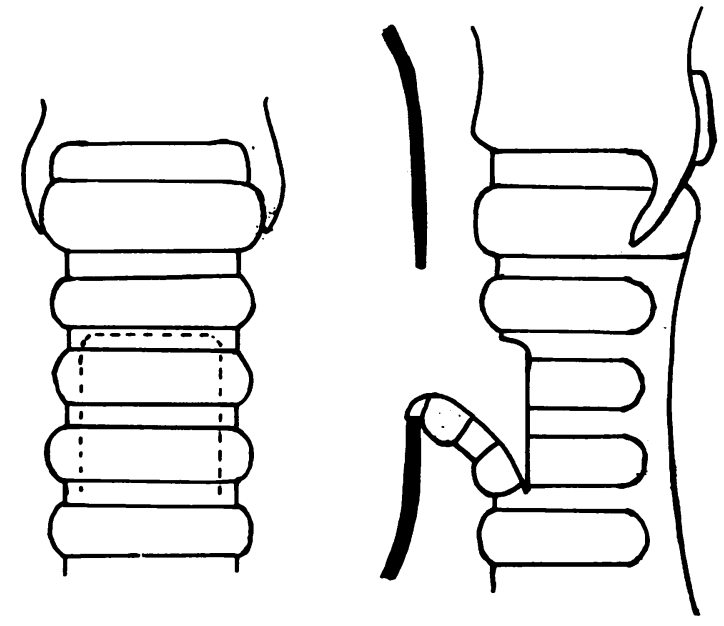

FIG. 4. Tracheostomy technique with attachment of the tracheal flap to skin.

was used in cases 1 and 3. There does not appear to be any relation between skin incision and stricture formation, but there is no doubt about the better cosmetic result following a transverse incision.

We would not now recommend the use of the flap procedure in children. It seems that contraction of scar tissue anterior to the trachea produces angling forwards of the relatively soft tracheal tissue at the stoma site. This angling plus the smaller tracheal lumen may be sufficient to produce significant narrowing accentuated by the development of granulation tissue and further increased by puckering of the membranous posterior tracheal wall. In case 4 the resulting deformity was similar to a double-barrelled colostomy, and a similar case was described by Clarke (1965).

Similar distortion has not been seen in the adult. This may be due to the firmer adult trachea or to its relatively wider lumen.

In the adult cases all the strictures seen were at the level of the cuff of the tracheostomy tube. Our present practice is to release the cuff for 5 minutes in every hour and to use the minimum cuff inflation necessary to secure absence of air leak. The largest possible size of tracheostomy tube should be used. We have noticed on several occasions in non-survivors of open-heart procedures that the tracheal mucosa is completely destroyed at the cuff site even when this practice is correctly followed. Gibson (1967) comments on the high incidence of tracheal damage. 
The flap procedure eliminates the danger of anterior tube displacement, permits of easier and safer reintroduction of tubes, and provides easy access to the bronchial tree for bronchoscopy. The tracheal window should be of the minimum size necessary. If this is exceeded there is a tendency for the lateral walls of the window to collapse into the trachea. In children there is disagreement as to whether the tracheal opening should consist of a vertical slit or a small window, the latter without a flap. Pressure necrosis of the edges of the slit after a tube has been in position for a few days produces a situation similar to a window, but with the disadvantage that there is necrotic tracheal tissue in the area where subsequent healing is to occur.

In our experience, tracheal strictures following tracheostomy have been of two types - the firm, fibrous, non-infected stricture developing over a period of time, and the early onset soft stricture occurring soon after removal of the tube and in which infection is probably present with granulation tissue adding to the tracheal narrowing. We have not been able to relate the presence of a specific infection to the subsequent development of stricture.

In the former group, excision with end-to-end anastomosis has given good results and has been possible in all our cases. Flavell in 1959 described this technique, and a further successful result was published by Binet and Aboulker (1961). Tracheal tension at the suture line may be reduced by using the $\mathrm{Z}$ plasty technique described by Narodick, Worman, and Pemberton (1965) and Worman, Starr, and Narodick (1966). Recurrent laryngeal nerve damage has occurred in one patient (case 6).

We have no experience of the staged plastic reconstruction (Gibson, 1967), though this may well have value in strictures longer than $2.5 \mathrm{~cm}$. Miscall, McKittrick, Giordano, and Nolan (1963) describe the excision of a 4-cm. stricture with end-to-end reconstruction, though extensive mediastinal mobilization was necessary through a sternal-splitting incision. Johnston et al. (1967) describe a conservative approach and recommend repeated tracheal dilatation as the treatment of choice. This may be true with the lesser degrees of narrowing, but with the tight strictures attempts at dilatation to introduce an endotracheal tube have been associated with bleeding. The patients are usually extremely frightened by their symptoms and have been immensely grateful for the restoration of a normal tracheal lumen.

Attempts at immediate reconstruction in the second type of stricture have failed in our hands. Naso-tracheal intubation across the area of narrowing would need to be for a time which would likely produce vocal cord damage. We believe that re-creation of tracheostomy through the damaged area is necessary with the introduction of a tracheostomy tube of the non-cuffed Portex Great Ormond Street pattern. This tube should be left in position for a minimum of three months to permit organization of healing tissues and eradication of infection. Repair is then carried out.

To leave a naso-tracheal plastic tube across the repair site is an attractive idea, but it has the disadvantage of vocal cord damage and irritation of the tracheal mucosa, and, most important, we have found that it is difficult to manage a child with a small-lumen tube. These tubes block easily, either by secretions or by becoming kinked at soft palate level, and often need to be replaced every 24 to 48 hours with full bronchial toilet under general anaesthetic at the same time. Facilities for immediate extubation and bronchoscopy must be present at all times. The provision of effective and adequate humidification is essential. Previous techniques of humidification have been unreliable and we have, as yet, insufficient experience with ultrasonic techniques to evaluate their properties.

\section{REFERENCES}

Binet, J. P., and Aboulker, P. (1961). Un cas de sténose trachéal, après trachéotomie, résection-suture de la trachée. Guérison. Mém. Acad. Chir., 87, 39.

Björk, V. O., and Engström, C. G. (1955). The treatment of ventilatory insufficiency after pulmonary resection with tracheostomy and prolonged artificial ventilation. J. thorac. Surg., 30, 356.

Clarke, D. B. (1965). Tracheostomy in a thoracic surgical unit. Thorax, $20,87$.

Flavell, G. (1959). Resection of tracheal stricture following tracheotomy, with primary anastomosis. Proc. roy. Soc. Med., 52, 143.

Fraser, K., and Bell, P. R. F. (1967). Distal tracheal stenosis following tracheostomy. Brit.J. Surg., 54, 302.

Gibson, P. (1967). Aetiology and repair of tracheal stenosis following tracheostomy and intermittent positive pressure respiration. Thorax, 22, 1 .

Johnston, J. B., Wright, J. S., and Hercus, V. (1967). Tracheal stenosis following tracheostomy-a conservative approach to treatment. J. thorac. cardiovasc. Surg., 53, 206.

Meade, J. W. (1961). Tracheostomy-its complications and their management. New Engl. J. Med., 265, 519.

Miscall, L., McKittrick, J. B., Giordano, R. P., and Nolan, R. B. (1963). Stenosis of trachea, resection and end to end anastomosis. Arch. Surg., 87, 726.

Narodick, B. G., Worman, L. W., and Pemberton, A. H. (1965). Relaxation technique for tracheal reconstruction. Ann. thorac. Surg., 1, 190.

Putney, F. J. (1955). Complications and postoperative care of tracheotomy. Arch. Otolaryng., 62, 272.

Watts, J. McK. (1963). Tracheostomy in modern practice. Brit. J. Surg., 50, 954.

Worman, L. W., Starr, C., and Narodick, B. G. (1966). Tracheoplasty technics for reconstruction after extensive resection. Amer. J Surg., 111, 819. 